their frequent high risk exposures. HIV transmission among PWID appears to be occurring at a rapid pace. Essential interventions are needed to slow transmission.

\section{P3.098 TUBERCULOSIS AMONG HIV POSITIVE CASES IN UMN HOSPITAL TANSEN -WESTERN NEPAL}

doi:10.1136/sextrans-2013-051184.0557

1.2J R Dhungana, ${ }^{3 P}$ Ghimire, ${ }^{4} \mathrm{D}$ S Bam, ${ }^{5 B}$ Rijal. 'TriChandra College, PO Box 690 Kathmandu, Nepal; ' $N e$ palese Association of Medical Microbiology(NAMM), PO Box 690, Kathmandu Nepal, Nepal; ${ }^{3}$ Central Department of Microbiology, TU, Kathmandu, Nepal; ${ }^{4}$ SAARC TB Centre/National TB Center, Bhaktapur, Nepal; ${ }^{5}$ OMM, TU, Kathmandu, Nepal

Background Human Immunodeficiency Virus infection has become a major public health problem worldwide. Tuberculosis (TB) is one of the most opportunistic infection (OI) among HIV infected patient. This study was conducted to study the prevalence of Opportunistic Infection TB among HIV positive patients visiting UMN Hospital Tansen, Palpa.

Methods HIV cases were diagnosed on the basis of rapid test kit HIV TRI DOT. TB cases were diagnosed on the basis of AFB staining, cultural methods, positive tuberculin test, radiographic abnormalities, and positive response to anti-tuberculous drugs.

Results A total of 8510 suspected HIV were included for the study. Of which, $81(0.95 \%)$ were found HIV positive. Out of $81 \mathrm{HIV}$ infected patients, 80\% (65/81) were Hospital OPD Patients and 16 $(20 \%)$ were Hospital admitted In-Patient. Among $81 \mathrm{HIV}$ infected patients, 28 patients were diagnosed as TB infection and 53 were without tuberculosis infection. Eighty six percent of $\mathrm{TB}$ cases were pulmonary tuberculosis (PTB) and $14 \%$ were extra-pulmonary tuberculosis. Out of $28 \mathrm{Co}$-infected HIV/TB cases, $75 \%$ were male and $25 \%$ were female. Of the $24 \mathrm{HIV} / \mathrm{PTB}$ Co-infected cases, 16 $(66.6 \%)$ were sputum AFB positive pulmonary tuberculosis and $33.3 \%(8 / 24)$ were sputum AFB negative pulmonary tuberculosis. Out of $4 \mathrm{HIV} /$ Extra-PTB co-infected patient,75\%(3/4) were TB Meningitis and $25 \%(1 / 4)$ were Miliary TB, in which 3 male patients and a female patient. Conclusion: Infection of $\mathrm{TB}$ among HIV infected patients of this study were ranged in age from 25 years50 years.

Key Words HIV, Opportunistic Infection (OI), Tuberculosis (TB), Pulmonary TB, Extra-PTB, AFB Positive, Tuberculous Meningitis (TBM), Miliary TB,

\section{P3.099 SEX WORK AS AN EMERGING RISK FACTOR FOR HIV SEROCONVERSION AMONG INJECTION DRUG USERS IN THE SURVUDI NETWORK}

doi:10.1136/sextrans-2013-051184.0558

${ }^{1} \mathbf{K}$ Blouin, ${ }^{2} \mathrm{P}$ Leclerc, ${ }^{2} \mathrm{C}$ Morissette, ${ }^{3} \mathrm{E}$ Roy, ${ }^{4} \mathrm{C}$ Blanchette, ${ }^{1} \mathrm{R}$ Parent, ${ }^{5} \mathrm{~B}$ Serhir, ${ }^{4} \mathrm{M}$ Alary, the SurvUDI Working Group. 'Institut national de santé publique du Québec, Unité des infections transmissibles sexuellement et par le sang, Québec, OC, Canada, 2Direction de Santé Publique de l'Agence de la santé et des services sociaux de Montréal, Montréal, OC, Canada; ${ }^{3}$ Université de Sherbrooke à Longueuil, Département des Sciences de la Santé Communautaire, Longueuil, OC, Canada; ' Université Laval, Unité de Recherche en Santé des Populations/Centre de recherche du CHU de Québec, Québec, OC, Canada; ${ }^{5}$ Institut national de santé publique du Québec, Laboratoire de santé publique du Québec, Sainte-Anne de Bellevue, OC, Canada

Background Previous analyses of SurvUDI data have shown an emerging positive association between sex work and HIV incidence among injection drug users (IDUs).

Objective To characterise the association between sex work and HIV seroconversion among IDUs in the SurvUDI network between 2004 and 2010.

Methods Participants who had injected in the past 6 months were recruited across the Province of Quebec and Ottawa, Canada, mainly in harm reduction programmes. They completed a questionnaire and provided saliva for HIV antibody testing. Multiple visits were linked through an encrypted identifying code. The association between sex work (defined as reporting at least one client sex partner in the last six months) and HIV seroconversion was tested with a Cox proportional hazards model. Time-dependent covariables tested as potential confounders were age ( $<25$ vs. $\geq 25$ years), sex, cocaine as the most often injected drug, injection with strangers, injection with a needle previously used by someone else, and consistent condom use (defined as always using a condom for vaginal and anal sex with casual, client and paid sex partners, with no sex partners as reference category). Covariables were retained as confounders when they changed the adjusted hazard ratio (AHR) by $>10 \%$ when removed from the complete model.

Results Sixty-four HIV seroconversions were observed during 3020 person-years of follow-up. In the final multivariate model, HIV incidence was significantly associated with sex work (AHR $=1.94,95 \%$ CI: $1.09-3.46, p<0.03)$ and injection with a needle previously used by someone else (AHR $=3.13,95 \% \mathrm{CI}: 1.88-5.22, \mathrm{p}<0.0001$ ), adjusted for age. Sex did not modify the association with sex work. Conclusion Sex work is independently associated with HIV incidence among IDUs. Further studies are needed to understand whether this association is related to sexual transmission or if sex work is an intermediate variable between other unknown vulnerability factors and HIV transmission.

\section{P3.100 FILLING THE KNOWLEDGE GAP: MEASURING HIV PREVALENCE AND RISK FACTORS AMONG POPULATIONS MOST VULNERABLE TO HIV IN LIBYA}

doi:10.1136/sextrans-2013-051184.0559

IJ J Valadez, 'S Berendes, 'C Jeffery, ${ }^{2} \mathrm{~J}$ Thomson, ${ }^{3} \mathrm{H}$ Ben Othman, 'S Moxon, ${ }^{2} \mathrm{~L}$ Danon, 'A A Turki, 'R Saffialden, 'L Mirzoyan. 'Liverpool School of Tropical Medicine, Liverpool, UK; ${ }^{2}$ University of Warwick, Mathematics Institute, Coventry, UK; ${ }^{3} \mathrm{National}$ Centre for Diseases Control, National AIDS Programme, Tripoli, Libyan Arab Jamahiriya

Background There has been no sound evidence on the status and dynamics of Libya's HIV-epidemic, which is urgently needed to inform near-term policy making while the window of opportunity to act is still open. With funding from the European Union we therefore aimed to assess HIV prevalence and related risk factors among populations most vulnerable to HIV.

Methods Using respondent-driven sampling, we conducted a cross-sectional survey among 328 people who inject drugs (PWID), 227 men having sex with men (MSM), and 69 female sex workers (FSW) in Tripoli. We collected behavioural data and blood samples for HIV, hepatitis C and B testing.

Results We estimated HIV-prevalence of $87 \%$ among PWID, 3\% among MSM, and 16\% among FSW. We detected high levels of IDUrelated, and sexual risk factors, in the context of strong stigma and lack of prevention programmes. Of particular concern, $85 \%$ of PWID reported having shared needles, only $21 \%$ of MSM used a condom at last sex, and $28 \%$ of FSW experienced STI symptoms during the last year. Over a third of MSM had risky sex with men and women and nearly a third of FSW had 50 or more sexual partners in the past six months.

Conclusion In this first bio-behavioural survey in Libya we detected among PWID one of the highest (or even the highest) levels of HIV-infection worldwide in the absence of a comprehensive harm-reduction programme. There is urgent need to implement an effective National HIV-Strategy informed by the results of this research, and designed to account for the overlap of high-risk sexual and drug-using networks. The risk of further transmission within different risk groups and to the general population is particularly high given the recent military events that led to increased violence, migration, and the disruption of essential HIV-related services and structures. 